\title{
PRODUCTIVIDAD DE COCHINILLA DACTYLOPIUS COCCUS (HEMIPTERA: DACTYLOPIIDAE) EN CLADODIOS DE OPUNTIA FICUS-INDICA (CACTACEA) CON DIFERENTES TRATAMIENTOS DE FERTILIZACIÓN
}

\section{Violeta CORONADO-FLORES, ${ }^{1}$ Mario Alberto TORNERO-CAMPANTE, ${ }^{1}$ Ramón NÚÑEZ-TOVAR, ${ }^{1}$ José LUIS JARAMILLO-VILLANUEVA ${ }^{1}$ y SANTIAGO DE JESÚS MÉNDEZ-GALLEGOS ${ }^{2, *}$}

\author{
${ }^{1}$ Colegio de Postgraduados, Campus Puebla, km. 125.5. Carr. Fed. México-Puebla. C.P. 72760. Puebla, Puebla. \\ ${ }^{2}$ Colegio de Postgraduados, Campus San Luis Potosí. Agustín de Iturbide \# 73. Salinas de Hidalgo, San Luis \\ Potosí. México.<jmendez@colpos.mx>
}

Recibido: 30/05/2014; aceptado: 09/01/2015

\begin{abstract}
Coronado-Flores, V., Tornero-Campante, M. A., Núñez-Tovar, R., Jaramillo-Villanueva, J. L. \& Méndez-Gallegos, S. de J. 2015. Productividad de cochinilla Dactylopius coccus (Hemiptera: Dactylopiidae) en cladodios de Opuntia ficus-indica (Cactacea) con diferentes tratamientos de fertilización. Acta Zoológica Mexicana (n. s.), 31(2): 183-189.
\end{abstract}

RESUMEN. Para determinar la influencia del contenido nutrimental y características anatómicas de cladodios de nopal (Opuntia ficus-indica L. Mill.) provenientes de cuatro tratamientos de fertilización química y orgánica sobre el rendimiento de cochinilla Dactylopius coccus, con respecto al número de hembras y su peso seco, así como de su calidad, en función del contenido de ácido carmínico, se estableció un estudio en condiciones de invernadero en el estado de Morelos. Aquellos cladodios a los que se les adicionó solo fertilización química generaron el mayor número de hembras y peso seco; mientras que los insectos criados sobre cladodios a los que se les aplicó fertilización química-orgánica presentaron los valores mayores de ácido carmínico. El contenido de oxalatos de calcio en el cladodio y el grosor de la cutícula influyeron negativamente en el número de hembras por cladodio. Se registró una correlación positiva significativa entre el contenido de nitrógeno en el cladodio y el número de hembras; no obstante, el nitrógeno influyó negativamente en el contenido de ácido carmínico. La fertilización previa a la que es sometida el nopal puede influir en el rendimiento y calidad de $D$. coccus.

Palabras clave: rendimiento, ácido carmínico, caracterización físicoquímica, cutícula, oxalatos.

\section{INTRODUCCIÓN}

La grana o cochinilla Dactylopius coccus Costa (Hemiptera: Dactylopiidae) (Gullan \& Cook 2001) parasita principalmente a especies de Opuntia y Nopalea (Flores-Flores \& Tekelenburg 1995). Su importancia económica y social radica en que de los cuerpos secos de las hembras adultas se obtiene un colorante natural conocido como acido carmínico (AC), empleado en la industria textil, farmacéutica, de cosméticos y alimentaria (Méndez-Gallegos et al. 2003; Tovar et al. 2005). El AC es uno de los pocos colorantes naturales avalados por la Organización Mundial de la Salud en Estados Unidos de América (FAO/OMS 2000) y por la Comunidad Europea para ser utilizado en
Coronado-Flores, V., Tornero-Campante, M. A., Núñez-Tovar, R., Jaramillo-Villanueva, J. L. \& Méndez-Gallegos, S. de J. 2015. Productivity of cochineal insects Dactylopius coccus (Hemiptera: Dactylopiidae) reared on cactus pear cladodes Opuntia ficus-indica (cactacea) produced under different fertilization conditions. Acta Zoológica Mexicana (n. s.), 31(2): 183-189.

ABSTRACT. The yield, on the number of females and their dry weight and quality, depending on the content of carminic acid of Dactylopius coccus were determined based on the nutrient content and some anatomical features of cactus pear cladodes (Opuntia ficus-indica L. Mill.) from four treatments of chemical and organic fertilization in Morelos state. Cladodes that were added only chemical fertilizers generated the largest number of females and, therefore, a higher dry weight; while insects raised on cladodes, to which organic-chemical fertilization was applied, showed higher values of carminic acid. The calcium oxalate content in cladode, and cuticle thickness negatively affected the number of females per cladode. A significant positive correlation between the content of nitrogen in the cladode and the number of females was recorded; however, it had a negative influence on the content of carminic acid. Previous cactus fertilization can influence the yield and quality of D. coccus.

Key words: yield, carminic acid, physico-chemical characterization, cuticle, oxalates.

alimentos (European Union 1989; European Union 1994). El contenido de AC es el principal parámetro usado para determinar la calidad comercial del insecto y su precio de venta. Esta característica es la razón fundamental por la que $D$. coccus tiene un valor mayor en comparación con la cochinilla silvestre (Dactylopius spp.), cuya concentración de AC es menor (Flores-Hernández et al. 2006).

Considerando las perspectivas económicas que la cría comercial de $D$. coccus puede representar como alternativa de producción para los habitantes del medio rural en México, durante los últimos años se han desarrollado trabajos de investigación para seleccionar los cultivares mas adecuados para su cría (Tovar et al. 2005; Méndez-Gallegos et al. 2010); para diseñar y evaluar diversos sistemas 
de cría intensiva a nivel comercial (Campos-Figueroa \& Llanderal-Cázares 2003; Aldama-Aguilera \& Llanderal 2003; Aldama et al. 2005); determinar los factores limitantes de su producción (Méndez-Gallegos et al. 1993; Montiel 1995; Aldama et al. 2005; Esparza et al. 2008); conocer aspectos de su morfología y citología (Ramírez-Cruz et al. 2008; Caselín-Castro et al. 2010; Ramírez-Cruz 2012) y caracterizar su perfil cromatográfico (Chávez-Moreno et al. 2010), entre otros estudios.

No obstante, poco se conoce acerca de la influencia de la condición nutricional de los cladodios sobre el rendimiento y calidad de D. coccus. Tekelenburg (1995) afirma que la fertilización orgánica favorece el establecimiento y calidad del insecto; mientras, Vigueras et al. (1993) señalan que el nitrógeno, azufre y el calcio son innecesarios para la producción de cochinillas adultas; Tovar et al. (2008) afirman que la concentración de nitrógeno y zinc favorecen el rendimiento del insecto. En un estudio posterior, Vigueras \& Portillo (1995) determinaron que la presencia de azufre, y la ausencia de potasio, nitrógeno y microelementos en el cladodio tienen efectos positivos sobre el contenido de AC; por lo que los resultados obtenidos hasta el momento son poco claros y contradictorios.

Considerando que un incremento en la rentabilidad y calidad de la cochinilla mexicana permitirá competir en los mercados internacionales con mayores ventajas, en el presente estudio se evalúo el contenido de nitrógeno, potasio, fósforo, azufre, calcio, fierro, zinc, magnesio y sodio, así como el grosor de cutícula y el número de oxalatos de calcio en cladodios de nopal Opuntia ficus-indica L. (Mill.) provenientes de cuatro modalidades de fertilización sobre el rendimiento y contenido de ácido carmínico de $D$. coccus.

\section{MATERIALES Y MÉTODOS}

El trabajo experimental se realizó en condiciones semicontroladas en los invernaderos de la Sociedad de Producción Rural "Campo Carmín”. Las coordenadas de dichas instalaciones son 1845'17', N y 99¹1'37', W y se ubican en el municipio de Emiliano Zapata, estado de Morelos, México.
Obtención del material vegetal. Los cladodios de $O$. ficus-indica se colectaron en huertos donde se aplicaron diferentes tratamientos de fertilización: química $(\mathrm{Fq})$, orgánica (Fo), química-orgánica (Fqo) y sin aplicación de fertilizante (sF) (Cuadro 1). Los cladodios seleccionados fueron de seis meses de edad, turgentes, sin daños por plagas y enfermedades, y con un tamaño aproximado de 40 por $20 \mathrm{~cm}$ de largo y ancho, respectivamente.

Sistema de producción. El sistema de cría empleado fue el de "penca colgada invertida" (cladodio en suspensión) en invernadero. El pie de cría inicial del insecto fue provisto por la Sociedad de Producción Rural "Campo Carmín”. El método de infestación utilizado fue el de "penca infestadora”. Durante el desarrollo del experimento, los cladodios fueron monitoreados constantemente y, cuando fue preciso, la cochinilla silvestre fue eliminada. También, los problemas de pudrición fueron controlados, mediante la desinfección previa de los ganchos con hipoclorito de sodio (Cloralex ${ }^{\circledR}$ ) 5\% y caldo bordelés. La cosecha se realizó por arrastre, mediante una brocha. La cochinilla cosechada se limpió manualmente utilizando pinzas de disección para eliminar restos de capullos, secreción cerosa, ninfas, machos, entre otros.

Se cuantificaron las hembras adultas en oviposición y posteriormente se obtuvo el peso fresco y peso seco (g), utilizando una balanza analítica OHAUS ${ }^{\circledR} \#$ EP413-4A.

La muerte y secado del insecto se realizaron en el interior del invernadero utilizando una modificación del método de muerte natural a la sombra post-oviposición (Méndez-Gallegos 2001).

Determinación del contenido de ácido carmínico. La extracción y cuantificación del contenido de AC se realizaron por triplicado utilizando la metodología propuesta por González et al. (2002). Inicialmente, los cuerpos secos de las hembras se molieron finamente en un mortero de cerámica. Luego, una cantidad de 0.125 g de cochinilla molida se disolvió en $30 \mathrm{~mL}$ de ácido clorhídrico $(\mathrm{HCl})$ $2 \mathrm{~N}$; sucesivamente, la mezcla se homogenizó durante un minuto en un agitador magnético marca Mistral-Magnestir Lab Line, modelo $1150 \AA$.

El AC fue extraído a baño de María (Clifton ${ }^{\circledR}$ SN 68054) a $65{ }^{\circ} \mathrm{C}$ durante $35 \mathrm{~min}$, temperatura y tiempo de máxima extracción de AC; posteriormente, los tubos se

Cuadro 1. Características de los tratamientos de fertilización.

\begin{tabular}{|c|c|c|}
\hline Tratamiento & Procedencia & Fertilización \\
\hline Fqo & Atlixco, Puebla & 11/2 t de urea ha $\mathrm{haño}^{-1} \mathrm{y}$ estiércol vacuno dos veces al año. \\
\hline Fo & Emiliano Zapata, Morelos & $\begin{array}{l}1 \text { L NUTRIPRO forte }{ }^{\circledR} \text { en } 200 \mathrm{~L} \text { de agua } \text { ha }^{-1} \text { mes }^{-1} \text {; además } \\
\text { de una aplicación anual de estiércol vacuno y gallinaza. }\end{array}$ \\
\hline $\mathrm{Fq}$ & Atlixco, Puebla & $1 \frac{1}{2} \mathrm{t}_{\text {urea }} \mathrm{ha}^{-1} \mathrm{año}^{-1}$. \\
\hline $\mathrm{sF}$ & Villanueva, Zacatecas & Ninguna. \\
\hline
\end{tabular}


enfriaron durante $10 \mathrm{~min} \mathrm{y}$, finalmente, las muestras se centrifugaron a $7000 \mathrm{rpm}$ a $4{ }^{\circ} \mathrm{C}$ durante $15 \mathrm{~min}$ (centrífuga Fisher Scientific Marathon ${ }^{\circledR} 2100$ R). Se realizaron dos extracciones para estimar la cantidad de AC a $\lambda=494$ nm (Spectronic Instruments ${ }^{\circledR}$ modelo 4001 /4), espectro de máxima absorción del AC de acuerdo a González et al. (2002). Antes de cada lectura se utilizó un blanco de ácido clorhídrico $2 \mathrm{~N}$.

Análisis químico de tejido vegetal. Para conocer el estado nutricional de los cladodios de nopal se determinaron las concentraciones de N, K, P, S y Ca, considerado como elementos determinantes de la calidad del AC, de acuerdo con Vigueras \& Portillo (1995). Adicionalmente, se determinó la concentración de Fe, Zn, Mg y Na. La metodología de digestión húmeda con mezcla de doble ácido se usó para los elementos de N, K, P y Ca; para el caso del S se empleó el método de digestión ácida simple. Los análisis químicos de las muestras se realizaron en el Laboratorio de Nutrición Vegetal del Colegio de Postgraduados, Campus Montecillo.

Grosor de cutícula. La variable anatómica grosor de cutícula $(\mu \mathrm{m})$ se registró de acuerdo al método propuesto por Tovar et al. (2006). Para ello, se consideraron cuatro muestras de $4 \mathrm{~cm}^{2}$ de cada cladodio. A cada muestra se le hicieron cuatro cortes transversales finos. Las cuatro submuestras resultantes se colocaron en cajas Petri con etanol al $70 \%$ por $1 \mathrm{~h} y$, posteriormente, se introdujeron a hidróxido de potasio al $10 \%$ por $3 \mathrm{~h}$; posteriormente se observaron en microscopio óptico (objetivo 10X/ 0.25 ACHRO160/0.17). Las mediciones de cutícula se realizaron utilizando un ocular micrométrico $10 \mathrm{XM}$, que se calibró con un micrómetro objetivo.

\section{Determinación de oxalatos de calcio}

La cuantificación de oxalatos de calcio se realizó mediante la metodología propuesta por Tovar-Puente et al. (2007). Las determinaciones del número de cristales de oxalatos de calcio por campo se realizaron utilizando muestras de $4 \mathrm{~cm}^{2}$ de la parte media de cada cladodio. Se utilizaron cuatro cladodios de cada tratamiento de fertilización como repeticiones.

Diseño experimental. Se utilizó un diseño en bloques completos al azar para reducir y controlar la varianza del error experimental que ocasiona tener el experimento en estantes con diferentes alturas, y para tener mayor precisión al realizar la comparación entre los tratamientos. Los bloques estuvieron representados físicamente por tres niveles de altura: $0.5 \mathrm{~m}, 1 \mathrm{~m}, 1.5 \mathrm{~m}$ con respecto al suelo. Cada unidad experimental se conformó por cinco cladodios de nopal, con cuatro repeticiones por tratamiento; tanto los tratamientos como las repeticiones, se distribu- yeron completamente al azar. Las variables independientes fueron los tratamientos de fertilización de nopal; químico, orgánico, químico-orgánico y sin aplicación de fertilizante; y las dependientes, rendimiento de cochinilla, en función del número de hembras y su peso seco (g), y contenido de AC (\%).

Análisis estadístico. Se realizó un análisis de varianza (ANDEVA) y los efectos medios se compararon con la prueba de Duncan $(p \leq 0.01)$ para probar la hipótesis nula de igualdad de efectos (Villalpando et al. 2001). Asimismo, efectuó un análisis de correlación de Pearson para determinar el grado de asociaciones bivariadas entre la concentración inicial de elementos químicos presentes en los cladodios de nopal y el número de hembras, peso seco, y el contenido de AC de los tratamientos de fertilización. Los análisis estadísticos se realizaron mediante el procedimiento GLM del paquete estadístico SAS versión 9.3 para Windows (SAS, 2010).

\section{RESULTADOS Y DISCUSIÓN}

Influencia de cladodios de $\boldsymbol{O}$. ficus indica producidos en diferentes tratamientos de fertilización sobre el rendimiento de $\boldsymbol{D}$. coccus. Se encontró efecto altamente significativo de los tratamientos de fertilización previa del nopal sobre el número de hembras por cladodio ( $\mathrm{p}$ $\leq 0.01$ ). El número de insectos por cladodio fue mayor (785.6) en los cladodios provenientes de parcelas con fertilización química (Fq) $(p \leq 0.01)$, al de aquellos cladodios con fertilización orgánica (Fo), química-orgánico (Fqo) y sin fertilizante (sF); aunque entre los dos últimos tratamientos la diferencia estadística fue no significativa (Cuadro 2).

El número de hembras por cladodio en los cuatro casos, resultó superior a aquellos registrados por Tovar et al. (2005) y a los observados por Tekelenburg (1995), y Campos-Figueroa \& Llanderal-Cázares (2003), quienes obtuvieron 194, y entre 214 y 222 hembras por cladodio, respectivamente. La diferencia entre el número de hembras de los cladodios con fertilización orgánica y química-orgánica puede estar influida por la aplicación de

Cuadro 2. Influencia de la fertilización previa del nopal sobre el número de hembras adultas de cochinilla por cladodio.

\begin{tabular}{|c|c|}
\hline Tratamiento & Promedio de hembras cladodio ${ }^{-1}$ \\
\hline $\mathrm{Fq}$ & $785.6 \mathrm{a}$ \\
\hline Fo & $626.0 \mathrm{~b}$ \\
\hline Fqo & 411.1c \\
\hline $\mathrm{sF}$ & 366.0c \\
\hline
\end{tabular}

Medias con distinta letra son significativamente diferentes (Duncan a $p \leq 0.01)$. 
abono orgánico en forma de gallinaza en la modalidad de fertilización orgánica y de estiércol vacuno en la fertilización química-orgánica; ya que de acuerdo con Tekelenburg (1995), la fertilización orgánica tiene una influencia positiva sobre los niveles poblacionales de cochinilla, obteniendo mejores resultados con la aplicación de gallinaza y, en menor grado, con estiércol vacuno, porcino y composta.

El efecto de los tratamientos de fertilización previa del nopal sobre el peso seco (g) de la cochinilla por cladodio fue significativo $(\mathrm{p} \leq 0.01)$. Sin embargo, la diferencia estadística entre el peso de cochinilla producida en cladodios provenientes de los tratamientos orgánico y químico fue no significativa (Cuadro 3); las diferencias entre éstos

Cuadro 3. Peso seco promedio de hembras de cochinilla en cladodios provenientes de diferentes tratamientos de fertilización.

\begin{tabular}{ll}
\hline Tratamiento & Peso seco promedio \\
\hline (g) & \\
Fo & $1.72 \mathrm{a}$ \\
$\mathrm{Fq}$ & $1.66 \mathrm{a}$ \\
$\mathrm{sF}$ & $1.50 \mathrm{~b}$ \\
$\mathrm{Fqo}$ & $1.48 \mathrm{~b}$ \\
\hline
\end{tabular}

Medias con distinta letra son significativamente diferentes (Duncan a $p \leq 0.01)$ con el tratamiento sin fertilización o con el de fertilización química-orgánica sí fueron significativas. Todos los promedios obtenidos se encuentran dentro de los valores observados por Méndez (2001), quien registró valores de entre 1 y 2 g de cochinilla seca por cladodio y fueron superiores a los obtenidos por Tovar et al. (2005).

Este estudio demuestra que para fines prácticos de estimación de rendimientos en la cría comercial de la cochinilla, de acuerdo al número de hembras y peso seco obtenidos, y considerando aquellos cladodios sometidos a fertilización orgánica, se requieren 581 cladodios infestados para obtener un kilogramo de cochinilla seca; mientras que se requieren 23, 81 y 94 cladodios más de los tratamientos con Fq, sF y Fqo, respectivamente (Figura 1).

Influencia del estado nutricional de cladodios sobre el rendimiento de $D$. coccus. El número de hembras por cladodio presenta una asociación positiva significativa $(r$ $=.971 ; p \leq 0.05)$ con el contenido de nitrógeno en el tejido vegetal (Cuadro 4). Este resultado coincide con lo registrado por Tovar et al. (2008), quienes señalaron que los contenidos de zinc y nitrógeno en tejido vegetal están relacionados positivamente con la producción de cochinilla. En la presente investigación también se observó una correlación positiva ( $r=0.527)$ entre el contenido de zinc y el número de hembras por cladodio; sin embargo, es no significativa a $p \leq 0.05$.

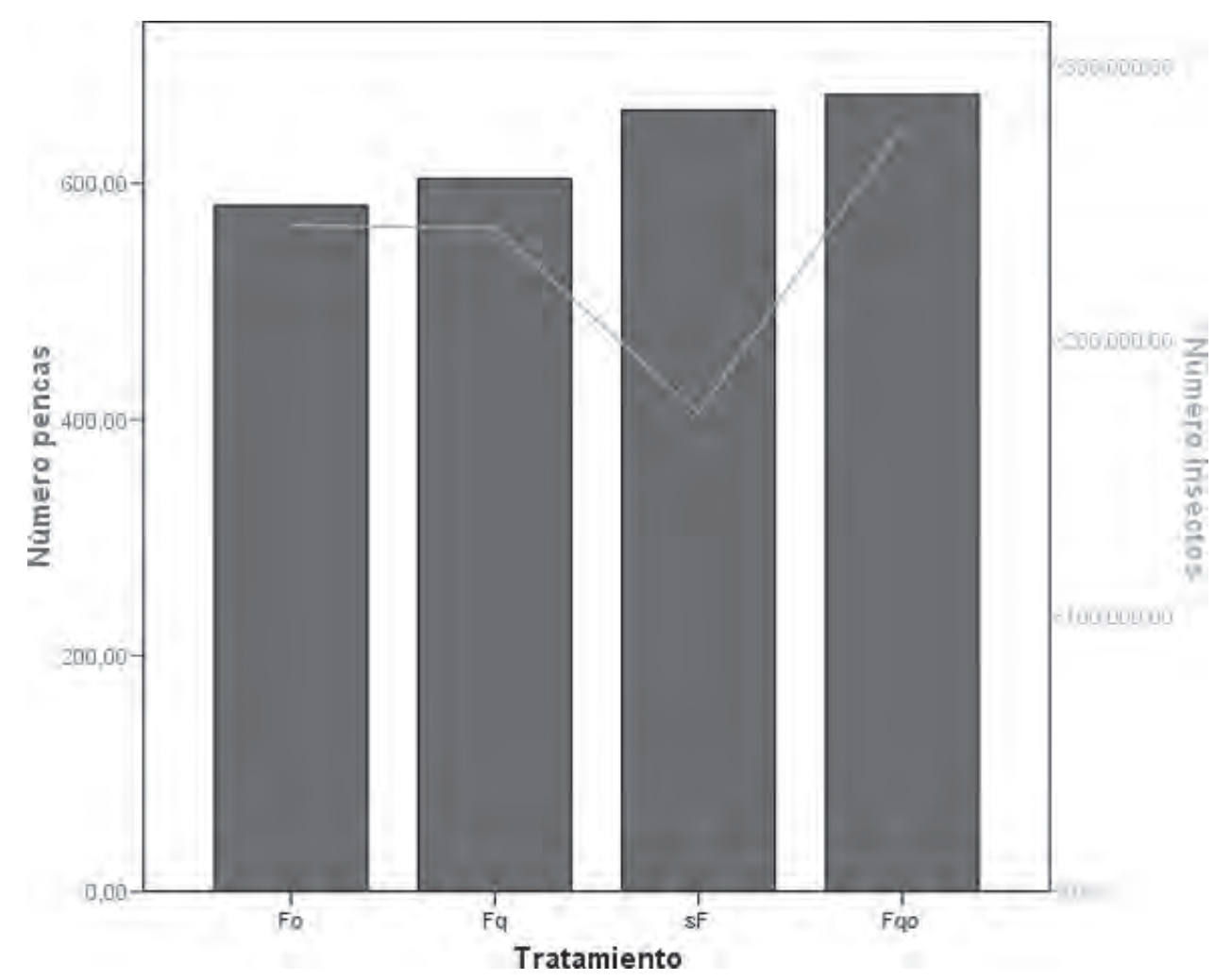

Figura 1. Requerimientos de cladodios de nopal e insectos deshidratados para obtener $1 \mathrm{~kg}$ de cochinilla seca. 
Cuadro 4. Concentración inicial de macro y micro elementos en cladodios de diferentes tratamientos de fertilización.

\begin{tabular}{lccccc}
\hline Elemento & & $\mathrm{Fq}$ & $\mathrm{Fqo}$ & $\mathrm{Fo}$ & $\mathrm{sF}$ \\
\hline $\mathrm{N}$ & $\%$ & 1.02 & 0.51 & 0.86 & 0.59 \\
$\mathrm{P}$ & $\mathrm{ppm}$ & 1467.0 & 587.7 & 1789.0 & 1477.1 \\
& $\%$ & 0.15 & 0.06 & 0.18 & 0.15 \\
$\mathrm{~K}$ & $\mathrm{ppm}$ & 19675 & 5766.8 & 11389 & 27179 \\
& $\%$ & 1.97 & 0.58 & 1.14 & 2.72 \\
$\mathrm{Ca}$ & $\mathrm{ppm}$ & 26746 & 12572 & 65637 & 43798 \\
& $\%$ & 2.67 & 1.26 & 6.56 & 4.38 \\
$\mathrm{Mg}$ & $\mathrm{ppm}$ & 23622 & 11105 & 37021 & 14391 \\
& $\%$ & 2.362 & 1.111 & 3.702 & 1.439 \\
$\mathrm{Fe}$ & $\mathrm{ppm}$ & 47.48 & 21.44 & 53.30 & 30.28 \\
& $\%$ & 0.005 & 0.002 & 0.005 & 0.003 \\
$\mathrm{Zn}$ & $\mathrm{ppm}$ & 88.5 & 48.3 & 147.1 & 72.6 \\
& $\%$ & 0.009 & 0.005 & 0.015 & 0.007 \\
$\mathrm{Na}$ & $\mathrm{ppm}$ & 277.52 & 222.75 & 308.74 & 240.42 \\
& & 0.028 & 0.022 & 0.031 & 0.024 \\
\hline
\end{tabular}

Se encontró correlación positiva significativa ( $p \leq$ $0.05)$ entre peso seco del insecto y los contenidos de magnesio $(r=0.959)$, fierro $(r=0.983)$ y sodio $(r=0.984)$ presentes en tejido vegetal. Esto significa que conforme aumentaron los contenidos de magnesio, fierro y sodio, también hubo un incremento en el peso seco de la cochinilla. Sin embargo, no fue posible encontrar evidencias en este sentido en la literatura, por lo tanto se sugiere realizar estudios más detallados que involucren estos microlementos.

Influencia del estado nutricional de cladodios sobre la calidad de $\boldsymbol{D}$. coccus. El contenido de AC de la cochinilla osciló entre 16.74 y $21.58 \%$ para la cochinilla criada en cladodios provenientes de los tratamientos de Fq y la Fqo, respectivamente (Cuadro 5). El contenido de AC de cochinilla cosechada en los cladodios provenientes de parcelas con tratamientos de Fqo, sF y Fo es mayor al mínimo requerido para ser considerada cochinilla de primera calidad, de acuerdo a las clasificaciones de la Fundación Boliviana Exporta y La Joya Eximport EIRL, quienes han establecido porcentajes de AC de 20\% y de 19 a 25\%, respectivamente. Por otro lado, la cochinilla proveniente de cladodios con fertilización química se ubica como cochinilla de segunda calidad, mayor a $12 \%$ y menor a 19\% de AC (http://www.lajoyaeximport.com/cochinilla. html). Los valores de AC obtenidos en esta investigación fueron superiores a los señalados por Méndez-Gallegos et al. (2010) pero inferiores a los registrados por Rodríguez et al. (2005).

Sólo la correlación negativa (-0.980) entre el porcentaje de AC y el nitrógeno resultó significativa $(p \leq 0.05)$.
Dicha tendencia concuerda con lo observado por Vigueras \& Portillo (1995) quienes mencionaron que la ausencia de nitrógeno, potasio y microelementos aumenta el porcentaje de AC.

Los cladodios provenientes de $\mathrm{Fq}$ presentaron el mayor nivel de nitrógeno con $1.02 \%$, pero este elemento tuvo un efecto detrimental sobre el contenido de AC (16.74\%). Los cladodios provenientes de Fo con 0.86\% de nitrógeno obtuvieron $19.18 \%$ de AC. Los cladodios sin fertilizante presentaron $0.59 \%$ de nitrógeno y $20.96 \%$ AC; mientras que aquellos cladodios con Fqo que tuvieron el nivel menor de nitrógeno $(0.51 \%)$ registraron la mayor concentración de AC con $21.58 \%$. Esto indica que a menor contenido de nitrógeno en el cladodio mayor es la concentración AC. Vale la pena destacar que cuando se planea producir cochinilla para ser utilizada como pie de cría, a mayor contenido de nitrógeno, el número de hembras por cladodio se incrementa.

Grosor de cutícula y oxalatos de calcio. El tipo de fertilización previa a que son sometidos los cladodios influye significativamente sobre el grosor de cutícula $(p \leq 0.01)$. Las diferencias entre el grosor de cutícula permitieron conformar dos grupos claramente diferenciados (Figura 2). Por un lado, aquellos cladodios procedentes de los tratamientos con Fo y sF presentaron una cutícula más gruesa; y por otro lado, los cladodios donde se utilizó fertilización química y química-orgánica tuvieron cutícula más delgada. El grosor de cutícula de los cladodios es mayor a los registrados por Tovar et al. (2008) quienes encontraron valores promedio de entre 7.83 y $14.08 \mu \mathrm{m}$ para quince variedades de nopal; asimismo, el grosor de cutícula de todos los tratamientos se encuentran dentro de los valores determinados por Nobel (2003) de 5 a $30 \mu \mathrm{m}$.

Se encontró efecto significativo de los tratamientos previos de fertilización del nopal sobre número de cristales de oxalatos de calcio $(p \leq 0.01)$. El número de cristales de oxalatos de calcio, presentó una tendencia similar a grosor de cutícula, ya que la diferencia entre los tratamientos Fo y sF fue no significativa, pero sí de éstos con respecto a la Fqo y a la Fq. El promedio más alto se registró en cladodios provenientes del tratamiento de Fo (34.6 cristales) y la menor (22.0 cristales) por campo correspondió a la Fq. Tovar-Puente et al. (2007) obser-

Cuadro 5. Ácido carmínico (AC) presente en D. coccus, criada en cladodios producidos con diferentes tratamientos de fertilización.

\begin{tabular}{lc}
\hline \multicolumn{1}{c}{ Tratamiento de fertilización } & $\%$ AC \\
\hline Fqo & 21.58 \\
sF & 20.96 \\
Fo & 19.18 \\
Fq & 16.74 \\
\hline
\end{tabular}




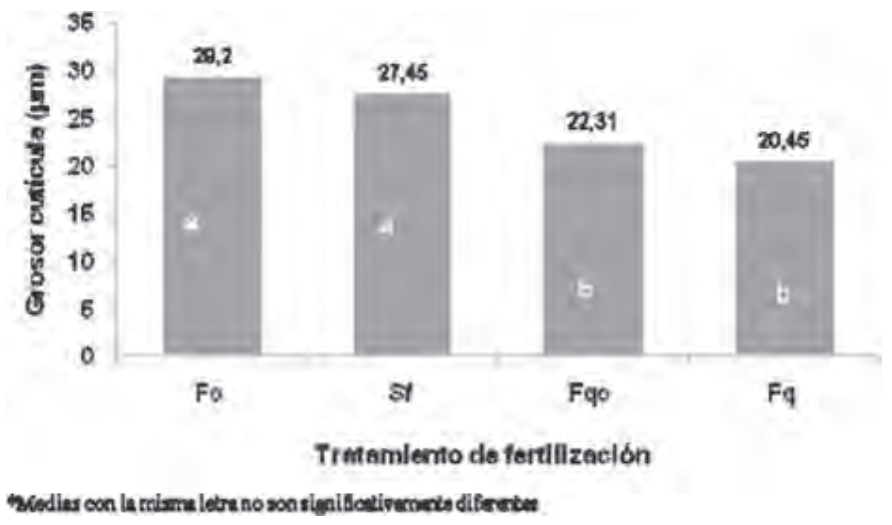

Figura 2. Grosor de cutícula $(\mu \mathrm{m})$ de cladodios producidos en diferentes modalidades de fertilización.

varon entre 17.5 y 56.8 cristales en quince variedades de nopal.

Se registró una correlación negativa $(r=-0.343)$ entre el grosor de cutícula de los cladodios y el número de hembras por cladodio. A su vez, el grosor de cutícula presentó una correlación positiva significativa $(r=0.984$; $p$ $\leq 0.01$ ), con el contenido de oxalatos de calcio; es decir, conforme aumenta el grosor de la cutícula también aumenta el contenido de oxalatos de calcio. Este aspecto reviste especial importancia y debe ser considerado en la producción comercial del insecto, ya que influye negativamente sobre el número de hembras por cladodio. De acuerdo con Méndez-Gallegos et al. (2003) la presencia y grosor de una capa de oxalatos de calcio en la pared de las células epidermales, posiblemente impiden la inserción de estiletes mandibulares del insecto, evitando con ésto su anclaje y posterior alimentación.

\section{CONCLUSIONES}

Este estudio no permitió generar una recomendación común de fertilización para la cría intensiva de cochinilla; mientras en aquellos cladodios provenientes de fertilización química registraron el mayor número de hembras y como consecuencia un mayor peso seco de cochinilla; en aquellos cladodios a los que se les aplicó fertilización química-orgánica presentaron los valores mayores de ácido carmínico.

El contenido de nitrógeno en el cladodio influyó positivamente sobre el número de hembras; no obstante, este elemento afectó negativamente el contenido de ácido carmínico.

AGRADECIMIENTOS. Al Consejo Nacional de Ciencia y Tecnología, al Colegio de Postgraduados Campus Puebla, a la Sociedad de Producción Rural Campo Carmín, a la Facultad de Ciencias Químicas y a la Escuela de Biología de la Benemérita Universidad Autónoma de
Puebla quienes apoyaron y brindaron facilidades para la realización de la presente investigación.

\section{LITERATURA CITADA}

Aldama-Aguilera, C. \& Llanderal-Cázares, C. 2003. Grana cochinilla: comparación de métodos de producción en penca cortada. Agrociencia, 37: 11-19.

Aldama A., Llanderal C. C., Soto H. M. \& Castillo M., L. E. 2005. Producción de grana cochinilla (Dactylopius coccus Costa) en plantas de nopal a la intemperie y en microtúneles. Agrociencia, 39: 161-171.

Campos-Figueroa, M. \& Llanderal-Cázares, C. 2003. Producción de grana-cochinilla Dactylopius coccus (Homóptera: Dactylopiidae) en invernadero. Agrociencia, 37: 149-155.

Caselín-Castro, S., Llanderal-Cázares, C., Méndez-Gallegos, S. de J., Ramírez-Cruz, A. \& Hernández-Hernández, F. 2010. Hemocytes of the cochineal insect: Ultraestructure. Archives of Insect Biochemistry and Physiology, 73: 176-192.

Chávez-Moreno, C. K., Tecante, A., Fragoso-Serrano, M. \& Pereda-Miranda, R. 2010. Metaboling profiling of Dactylopius (Hemiptera: Dactylopiidae) species pigment by geographical origin and hosts using multivariate data analysis. Bioechemical Systematics and Ecology, 38: 671-679.

Esparza G., G., Vigueras G., A. L. \& L. Portillo. 2008. Salpingogaster cochinellivora Guerin- meneville (Sirphidae) entomófago de Dactylopiidae y su confusión con los géneros Baccha sp. y Allograpta sp,. pp. 46-49. In: C. Llanderal C., D. H. Zetina, A. L. Vigueras G. \& L. Portillo (Eds.). Grana Cochinilla y Colorantes Naturales. Colegio de Postgraduados. México.

European Union (EU). 1989. Community Directive 89/107/EEC; Official Journal of the European Union. L 40; European Community: Brussels, Belgium,

European Union (EU). 1994. Community Directive 94/36/EEC; Official Journal of the European Union. L 237/13; European Community. Brussels, Belgium,

FAO/OMS Comité Mixto de Expertos en Aditivos Alimentarios (JECFA). 2000. Base de datos sobre aditivos alimentarios (con excepción de los utilizados como aromatizantes). http://www.codexalimentarius.net/web/jecfa.jsp

Flores-Flores, V. \& Tekelenburg, A. 1995. Dacti (Dactylopius coccus Costa) dye production. pp. 167-185. In: G. Barbera, P. Inglese \& E. Pimienta-Barrios (Eds.). Agro-ecology, cultivation and uses of cactus pear. FAO Plant Production and Protection Paper 132.

Flores-Hernández A., Murillo-Amador, B., Rueda-Puente, E. O., Salazar-Torres, J. C., García-Hernández, J. L. \& Troyo-Diéguez, E. 2006. Reproducción de cochinilla silvestre Dactylopius opuntiae (Homóptera: Dactylopiidae). Revista Mexicana de Biodiversidad, 77: 97-102.

González, M., Méndez, J., Carnero, A., Lobo, M. G. \& Alfonso, A. 2002. Optimizing conditions for the extraction of pigments in cochineals (Dactylopius coccus Costa) using response surface methodology. Journal of Agricultural and Food Chemistry, 50: 6968-6974.

Gullan, P. J. \& Cook, L. G. 2001. Are cochineal insects erioccids?. Entomologica Bari, 33: 91-99.

http://www.lajoyaeximport.com/cochinilla.html Octubre 2012.

Méndez-Gallegos, S. de J., Vera-Graziano, J., Bravo-Mójica, H. \& López-Collado, J. 1993. Tasas de supervivencia y reproducción de la grana-cochinilla Dactylopius coccus Costa (Homoptera: 
Dactylopiidae) a diferentes temperaturas. Agrociencia Serie Protección Vegetal, 4: 7-22.

Méndez G., S. J. 2001. Cultivo y manejo de la grana cochinilla. pp. 69-78. In: C. Llanderal C. \& R. Nieto H. (Eds.). Cría de la grana cochinilla para la producción de su pigmento. Colegio de Postgraduados. México.

Méndez-Gallegos, S. de J., Panzavolta, T. \& Tiberi, R. 2003. Carmine cochineal Dactylopius coccus Costa (Rhynchota: Dactylopiidae): Significance, production and use. Advances in Horticultural Science, 17: 165-171.

Méndez G., S. J. 2010. De los montes a la biofábrica: la evolución de los sistemas de cría de la cochinilla. pp. 135-144. In: L. Portillo \& A. L. Vigueras (Eds.). Conocimiento y aprovechamiento de la grana cochinilla. Universidad de Guadalajara. México.

Méndez-Gallegos, S. de J., Tarango-Arámbula, L. A., Carnero, A., Tiberi, R. \& Díaz-Gómez, O. 2010. Crecimiento poblacional de la cochinilla Dactylopius coccus Costa criada en cinco cultivares de nopal Opuntia ficus-indica Mill. Agrociencia, 44: 225-234.

Montiel, R. L. 1995. Morfología de Dactylopius coccus Costa (Homoptera: Dactylopiidae) y su biología y reproducción en dos fotoperiodos. Tesis de Maestría en Ciencias. Colegio de Postgraduados. Instituto de Fitosanidad. Programa de Entomología y Acarología. Montecillo, México. 104 p.

Nefzaoui A. \& Salem, H. B. 2003. Opuntia forraje estratégico y herramienta eficiente para combatir la desertificación en la región Wana. pp. 73-89. In: C. Mondragón-Jacobo \& S. Pérez-González (Eds.). El nopal (Opuntia spp.) como forraje. FAO Plant Production and Protection Paper 169.

Nobel, P. S. 2003. Ecofisiología de Opuntia ficus-indica. pp. 13-20. In: C. Mondragón-Jacobo C. \& S. Pérez-González (Eds.). El nopal (Opuntia spp.) como forraje. FAO Plant Production and Protection Paper 169.

Ramírez-Cruz, A., Llanderal-Cazáres, C. \& Racotta, R. 2008. Ovariole structure of the cochineal scale insect, Dactylopius coccus. Journal of Insect Science, 8: 1-5.

Ramírez-Cruz A. 2012. Ultraestructura del germario de Dactylopius coccus costa, 1829 (Hemiptera: Coccoidea: Dactylopiidae). Acta Zoológica Mexicana (n.s.), 28: 586-593.

Rodríguez L., C., Faúndez, E., Seymour, J., Escobar, C., Espinoza, L., Petroutsa, M., Ayres, A. \& Niemeyer, H. N. 2005. Factores bióticos y concentración del ácido carmínico en la cochinilla
(Dactylopius coccus Costa) (Homoptera: Dactylopiidae). Agricultura. Técnica, 65: 323-329.

SAS, Institute SAS System for Windows. V. 9.3. 2010. SAS Institute Inc., Cary, NC, 27513.USA.

Tekelenburg A. 1995. La producción de cochinilla (Dactylopius coccus Costa) en ambientes semicontrolados. pp. 48-55. In: E. Pimienta B., C. Neri L., A. Muñoz U. \& F. M Huerta M. (Eds.). Memorias del 6to Congreso Nacional y 4to. Internacional sobre el Conocimiento y Aprovechamiento del Nopal. Universidad de Guadalajara. Guadalajara, Jalisco, México.

Tovar, A., Pando-Moreno, M. \& C. Garza. 2005. Evaluation of three varieties of Opuntia ficus-indica (L.) Miller as hosts of the cochineal insect (Dactylopius coccus Costa (Homoptera: Dactylopiidae) in a semiarid area of northeastern Mexico. Economic Botany, 59: 3-7.

Tovar P., A., Pando-Moreno, M., González R., H., Vázquez A., R. E. \& M. A. Madrigal A. 2006. Caracterización química y física de quince cultivares de nopal de los géneros Opuntia y Nopalea. Bol. Nakari, 17: 29-35.

Tovar-Puente A., Pando-Moreno, M., González-Rodríguez, H., Scott-Morales, L. \& S. J. Méndez-Gallegos. 2007. Density of calcium oxalate crystals in 15 prickly pear cultivated species. Journal of the Professional Association for Cactus Development, 9: 91-98.

Tovar-Puente A., Pando-Moreno, M., González-Rodríguez, H., Scott-Morales, L. \& Méndez-Gallegos, S. J. 2008. Relación entre producción de grana (Datylopius coccus Costa) (Homoptera: Dactylopidae) y variables físicas y químicas del hospedante. pp. 62-66. In: C. Llanderal C., D. H. Zetina, A. L. Vigueras \& L. Portillo (Eds.). Grana cochinilla y colorantes naturales. Colegio de Postgraduados. México.

Vigueras G., A., Portillo L. \& Flores, V. 1993. Influencia de los macro y microelementos aplicados a cladodios de Opuntia ficus indica (L) Mill. sobre el desarrollo de la cochinilla. Quepo, Revista de la Sociedad Peruana de Cactus y Suculentas, 7: 81-91.

Vigueras G., A. \& Portillo, L. 1995. Determinación de ácido carmínico en dos generaciones de Dactylopius coccus Costa mediante cultivo hidropónico en nopal. Bol. Nakari, 6: 41-48.

Villalpando G., J., Castillo M., A., Ramírez G., M. E., Rendón S., G. \& Larqué S., M. U. 2001. Comparación de los procedimientos de Tukey, Duncan, Dunnett, Hsu y Bechhofer para selección de medias. Agrociencia, 35: 79-86. 